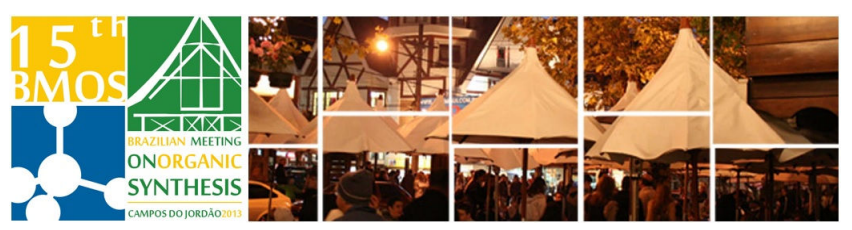

\title{
Intramolecular Aza-Anti-Michael Addition for the Synthesis of 2-Iminothiazolidines
}

\section{Misael Ferreira and Marcus Mandolesi Sá}

Universidade Federal de Santa Catarina, Departamento de Química, Florianópolis/SC, 88040-900 Brasil

*Corresponding author. Tel.: +55-48-37219967; fax: +55-48-37216850; e-mail: misaqmc@yahoo.com.br

Keywords: Allylic bromides, thioureas, 2-iminothiazolidines.

\section{INTRODUCTION}

Allylic bromides 1 (derived from the MoritaBaylis-Hillman adducts) are versatile intermediates for the preparation of cyclic compounds. ${ }^{1}$ In addition, substituted thioureas 2 are widely used in reactions with molecules having more than one electrophilic center allowing the synthesis of heterocycles with biological properties. $^{2}$

As part of our research interest in synthetic transformations involving allylic bromides 1 with ambident compounds, ${ }^{3}$ herein we report the intramolecular aza-anti-Michael reaction of allylic bromides 1 with thioureas 3 as a new methodology for the synthesis of 2-iminothiazolidine derivatives.

\section{RESULTS AND DISCUSSION}

Allylic bromides 1 were obtained in high yields by treating $\alpha$-methylene- $\beta$-hydroxyesters (Morita-BaylisHillman adducts) with $\mathrm{LiBr} / \mathrm{H}_{2} \mathrm{SO}_{4}$ in acetonitrile (75$90 \%$ yield). ${ }^{4}$

The reaction of allylic bromides 1 with $N$ substituted thioureas $\mathbf{2} \mathbf{a}$ in acetonitrile furnished isothiuronium salts $3 \quad(66-97 \%$, Scheme 1). Subsequent acetylation reactions of $N$-substituted salts 4 with acetic anhydride under basic medium at low temperature furnished a mixture of monoacetylated isomers ${ }^{3 \mathbf{b}}$ where $\mathbf{4}$ was the major product $\left(60-80 \%\right.$, conversion, determined by ${ }^{1} \mathrm{H}$ NMR $400 \mathrm{MHz}, \mathrm{CDCl}_{3}$, Scheme 1).

Monoacetylated isomer $4 a\left(\mathrm{R}_{1}=\mathrm{C}_{6} \mathrm{H}_{5}, \mathrm{R}_{2}=\mathrm{CH}_{3}\right)$ was purified by column chromatography. Subsequent treatment of $\mathbf{4 a}$ with base (DABCO) in acetonitrile allowed the unexpected formation of 2iminothiazolidine $\mathbf{6 a}$ through the conjugate addition at the $\alpha$-position of the alkenoate acceptor known as the anti-Michael reaction ( $91 \%$ yield). Complete structural elucidation of compound 6a was achieved by X-ray crystallography analysis (Scheme 1, Table 1). Cyclization reaction of crude mixtures of $N$ acetylated products 4 in basic medium without prior purification also furnished the corresponding 2iminothiazolidines 6 in reasonable yields (Scheme 1, Table 1).

In a more convergent synthetic approach, 2iminothiazolidines 6 could also be obtained in good yields from a one-step reaction of $N$-benzoyl- $N$ 'substituted thioureas $\mathbf{2 b}$ with allylic bromides $\mathbf{1}$ in the presence of DBU (Scheme 1, Table 2).

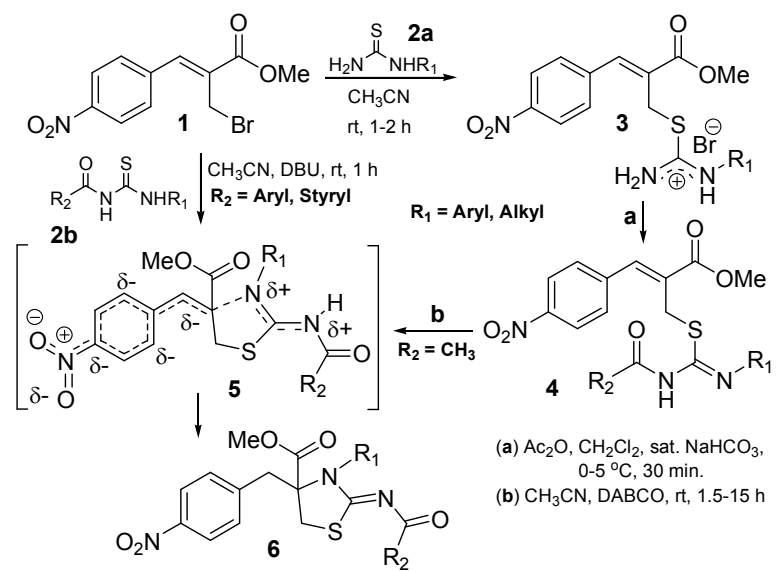

Scheme 1

Table 1. 2-Iminothiazolidines 6 from isothiouronium salts 3 .

\begin{tabular}{ccccc}
\hline $\mathbf{6}^{\mathbf{a}}$ & $\mathbf{\mathbf { R } _ { \mathbf { 1 } }}$ & Time (h) & Yield (\%) & $\mathbf{M p}\left({ }^{\mathbf{b}} \mathbf{C}\right)$ \\
\hline $\mathbf{6 a}$ & $\mathrm{C}_{6} \mathrm{H}_{5}$ & 1.5 & 73 & $151.5-153.0$ \\
$\mathbf{6 b}$ & $4-\mathrm{CH}_{3} \mathrm{C}_{6} \mathrm{H}_{4}$ & 4 & 47 & $174.5-176.0$ \\
$\mathbf{6 c}$ & $4-\mathrm{CH}_{3} \mathrm{OC}_{6} \mathrm{H}_{4}$ & 4 & 30 & $167.5-168.5$ \\
$\mathbf{6 d}$ & $\mathrm{CH}_{3}$ & 15 & 54 & $135.0-136.0$ \\
$\mathbf{6 e}$ & $\mathrm{CH}_{2}=\mathrm{CHCH}_{2}$ & 4.5 & 70 & $126.5-127.5$ \\
\hline${ }^{\mathrm{a}} \mathrm{R}_{2}=\mathrm{CH}_{3}{ }^{b}$ Isolated yield & & &
\end{tabular}

Table 2. 2-Iminothiazolidines 6 from allylic bromides 1.

\begin{tabular}{ccccc}
\hline $\mathbf{6}$ & $\mathbf{R}_{\mathbf{1}}$ & $\mathbf{R}_{\mathbf{2}}$ & Yield (\%) & $\mathbf{M p ~}\left(^{\circ} \mathbf{C}\right)$ \\
\hline $\mathbf{6 a}$ & $\mathrm{C}_{6} \mathrm{H}_{5}$ & $\mathrm{C}_{6} \mathrm{H}_{5}$ & 95 & $234.0-235.0$ \\
$\mathbf{6 b}$ & $\left(\mathrm{CH}_{3}\right)_{2} \mathrm{CH}$ & $\mathrm{C}_{6} \mathrm{H}_{5}$ & 84 & $131.5-133.5$ \\
$\mathbf{6 c}$ & $\mathrm{C}_{6} \mathrm{H}_{5} \mathrm{CH}_{2}$ & $4-\mathrm{CH}_{3} \mathrm{C}_{6} \mathrm{H}_{4}$ & 91 & $179.5-180.5$ \\
$\mathbf{6 d}$ & $\mathrm{CH}_{2}=\mathrm{CHCH}_{2}$ & $4-\mathrm{CH}_{3} \mathrm{C}_{6} \mathrm{H}_{4}$ & 88 & $141.0-142.0$ \\
$\mathbf{6 e}$ & $4-\mathrm{CH}_{3} \mathrm{C}_{6} \mathrm{H}_{4}$ & $\mathrm{C}_{6} \mathrm{H}_{5} \mathrm{CH}=\mathrm{CH}$ & 82 & $152.5-154.5$ \\
\hline${ }^{a}$ Isolated yield. & &
\end{tabular}

\section{CONCLUSION}

A simple protocol for the synthesis of 2iminothiazolidines $\mathbf{6}$ in goods yields via intramolecular aza-anti-Michael addition of MoritaBaylis-Hillman derivatives under mild conditions was developed.

\section{ACKNOWLEDGEMENTS}

\section{CAPES, CNPq, INCT-Catálise}

${ }^{1}$ Batra, S.; Singh, V. Tetrahedron 2008, 64, 4511.

(a) Gorczynski, M. J.; Leal, R. M.; Mooberry, S. L.; Bushweller, J. H.; Brown, M. L. Bioorg. Med. Chem. 2004, 12, 1029. (b) Jawale, D. V Pratap, U. R.; Rahuja, N.; Srivastava, A. K.; Mane, R. A. Bioorg. Med. Chem. Lett. 2012, 22, 436

${ }^{3}$ (a) Sá, M. M.; Fernandes, L.; Ferreira, M.; Bortoluzzi, A. J. Tetrahedron Lett. 2008, 49, 1228. (b) Sá, M. M.; Ferreira, M.; Bortoluzzi, A. J. Fernandes, L.; Cunha, S. Arkivoc 2010, xi, 303.

${ }^{4}$ Ferreira, M.; Fernandes, L.; Sá, M. M. J. Braz. Chem. Soc. 2009, 20, 564. $15^{\text {th }}$ Brazilian Meeting on Organic Synthesis - 15 ${ }^{\text {th }}$ BMOS - November 10-13, 2013 - Campos do Jordão, Brazil 